\title{
ZOONOSES IN BANGLADESH: THE ROLE OF VETERINARIAN IN PUBLIC HEALTH
}

\author{
M. Ariful Islam* \\ Department of Medicine, Faculty of Veterinary Science, Bangladesh Agricultural University \\ Mymensingh-2202, Bangladesh
}

\begin{abstract}
Zoonoses are diseases and infections that are naturally transmissible between vertebrate animals and man. Zoonoses may be categorized as emerging, reemerging and neglected zoonotic diseases which are widely prevalent and pose a great threat throughout the world including Bangladesh. Veterinary public health is a component of public health activities devoted to the application of professional veterinary skills, knowledge, and resources for the protection and improvement of public health. Many veterinarians are less aware of the importance of zoonoses than is desirable and medical clinicians who encounter zoonoses in human patients may either fail to recognize them or concentrate on treating the individual patient rather than disease control. This situation may be worsened where there is insufficient communication between veterinarians, the various health care professionals and public health organizations. However, the collaborative efforts between physicians and veterinarians in communication, sharing public health knowledge and research settings could do much to improve human and animal health. Much can be done by education, and in particular by increasing the awareness of different health professionals, and facilitating communication and collaboration between veterinary, public health and agricultural personnel. This will help us to approach and control zoonotic diseases in as efficient and effective a way as possible.
\end{abstract}

Key words: Veterinarian, public health, physician, collaboration, zoonoses, control, one health

\section{INTRODUCTION}

Zoonoses are diseases and infections that are naturally transmissible between vertebrate animals and man (WHO, 2006). They exert dual impacts on human population that are livestock dependent. This could be either through direct risk of infection by zoonoses or through reduced production by the livestock resulting in food insecurity and poverty (Molyneuxet al., 2011). It is estimated that over 600 million people worldwide are livestock-dependent, and represent up to $70 \%$ of the marginalized and poor population (FAO, 2004). Zoonotic diseases continue to represent an important health hazard in most parts of the world, where they cause considerable expenses and losses for the health and agricultural sectors. Even though the situation is improving in the industrialized world, zoonoses prevention and control will remain an area of major concern in most developing countries including Bangladesh.

Veterinary public health is a part of public health activities committed to the application of professional veterinary skills, knowledge, and resources for the protection and improvement of public health. There can be little doubt that the majority of veterinarians and human health professionals have a basic knowledge about zoonoses and have some theoretical understanding of the threat that they might pose to human health. But it is also apparent that in practice many health workers either fail to consider the possibility that they may be dealing with a zoonosis or ignore the public health implications of this type of infection. In addition, the significance or potential significance of zoonoses is often ignored by public health strategy and limited resources are available to investigate them. Veterinarians should play an important role to control and prevent zoonotic diseases. The main scientific and applied disease control areas where the unique knowledge and position of veterinary medicine in natural science has proved useful for the protection of human health are: foodborne diseases control-food safety, zoonoses control, communicable disease epidemiology and population medicine, comparative biomedicine, animal welfare, and environmental protection. This review focuses on role of veterinarians in controlling zoonoses as one of the most important components of veterinary public health with emphasis on a collaborative approach. However, closer collaborations are needed between veterinarians, physicians, and public health professionals in three areas viz. individual health, population health, and comparative medicine research for control of zoonotic diseases.

*Corresponding e-mail address: maislam77@gmail.com

Copyright (C) 2014 Bangladesh Society for Veterinary Medicine

All rights reserved 295/2014 


\section{Zoonoses in Bangladesh}

Zoonotic diseases are common throughout the world and constitute an important threat to human health in developing countries like Bangladesh. The results on the prevalence and effects of zoonotic diseases in humans and animals of Bangladesh are analyzed from the published literatures (Samad, 2011). It appears from the literature that there are about 1415 human pathogens of which $61 \%$ are zoonotic and nearly half of all human pathogens can be classified as emerging, of which $75 \%$ of these are caused by zoonotic pathogens. The major zoonotic bacterial diseases recorded in Bangladesh are Anthrax, Tuberculosis, Brucellosis, Salmonellosis, Campylobacteriosis and Leptospirosis, of which only Anthrax has been reported as clinical outbreaks form in both the humans and cattle (Samad, 2011). The major reported viral zoonotic diseases in Bangladesh include Avian influenza, Rabies, Nipah virus infection, Japanese encephalitis, Rotavirus and Dengue fever. Recently, Qfever $(6.38 \%)$ in domestic ruminants and brucellosis $(4.4 \%)$ in occupationally exposed people was reported (Rahman et al. 2012; Rahman et al. 2014) The major zoonotic parasites recorded in cats in Bangladesh include Paragonimuswestermani, Ancyclostomatubaeforme, Dirofilariaimmitis, Toxocaracati and Toxoplasma gondii, in dogs include Diphyllobothriumlatum, Diphylidiumcaninum, Echinococcusgranulosus, Ancylostomumcaninum, Gnathostomaspingerum,Toxocaracanis, Trichurisvulpis and in pigs include Entaemebeahistolytica and Balantidium coli. The prevalence of zoonotic protozoan diseases recorded in humans in Bangladesh includes amebiasis, giardiasis, cryptosporidiasis and visceral leishmaniasis (kala-azar). The review of Samad (2011) appears from the literatures that meat, milk, eggs, water and other foods contained high numbers of coliforms, staphylococci, salmonella pathogens. It appears from this review that all types of emerging, reemerging and neglected zoonotic diseases are widely prevalent and pose a great threat to human health in Bangladesh.

\section{Current status of medical and veterinary medical collaboration in Bangladesh}

In Bangladesh, unfortunately human and veterinary diseases have been treated as separate field and medical and veterinary medical does not communicate and or collaborate with each other. The educational background of physician and veterinary is different and their clinical practices is also separate. But, the collaboration between physician and veterinarian should be increased and diverse regarding human and animals health to control zoonoses. In Bangladesh, Institute of Public Health (IPH), National Institute of Preventive and Social Medicine (NIPSOM) are the national level public health institutes supporting in the different health policy formulation of the government and community health programs through research, training and services but they have no veterinarian. On the other hand, Institute of Epidemiology, Disease Control and Research (IEDCR) is the nation's focal point for conducting disease surveillance, outbreak investigation, research in the public health issue and training as well. Recently, Bangladesh was strike with some emerging diseases of animal origin like avian influenza, Anthrax and Nipah (Samad, 2011). IEDCR investigated all the outbreak cases in collaboration with Department of Livestock Services and International Centre for Diarrhoeal Disease Research, Bangladesh (ICDDR,B). But, it's really disappointing that there is no Veterinarians in the leading public health institutes in Bangladesh except ICDDR,B. Though, there are several veterinary institutions and medical education institute right now in Bangladesh, limited research is undertaken related to epidemiology of diseases as well as public health issue. Regional medical and veterinary education and research centre can collaborate and communicate information and research to combat against zoonoses. On the other hand, in the institutional level there is nothing associated with research that would provide a new insight into the epidemiology of diseases. That's why the Veterinarians, have poor base regarding the public health issues. As specialized Veterinarian is very few in the field of public health, proper steps should be taken to develop so that they could take the lead in the public health issue. Veterinary and medical institutes should include public health and veterinary public health course, respectively in their education system. Therefore, it is highly recommended that more research should be conducted related to public health as well as epidemiology of zoonotic diseases in order that upcoming Veterinarians might take the challenges of public health concern. Besides, it is strongly urge the government to recognize fully the necessity of direct veterinary contribution to public health policy development. In addition, more Veterinarians should be employed in the public health institute so that these institutes work independently by addressing the concerns outlined above. 


\section{Role of veterinarians in zoonoses control}

Veterinary public health is an important element of public health activities devoted to the application of professional veterinary skills, knowledge, and resources for the protection and improvement of public health. Veterinarians can play an important role globally in public health to control zoonoses including Bangladesh. Public health veterinarians can also work on issues such as, prevention and education of zonnoses, infectious disease outbreak investigations, food safety and protection, food-borne illness investigations, or environmental health issues, such as vector control or air or water quality, as well as occupational exposures. Veterinarians are accessible, expert sources of information regarding zonoonotic diseases and should be prepared to inform clients of risks specific to their communities (Lipton et al., 2008; Dvorak et al., 2008).

Poverty is thought to be one of the risk factors for zoonoses and food-borne diseases in both rural and urban consumers. Consumers should be provided with safe food but, unfortunately, livestock organization of Bangladesh has no such facilities and manpower to determine the presence of harmful agents in food. Public health veterinarians can work to improve food safety and eliminate food-borne zoonotic threats to reduce the burden of foodborne illnesses in the Bangladesh, as well as other countries. Using Veterinary epidemiology skills, many public health veterinarians work closely with human medical and public health professionals to help investigate foodborne disease outbreaks. The issues considered such as food safety, control of 'zoonotic' diseases, meat inspection and abattoir management are very poorly addressed in Bangladesh. Thus, veterinary and human health authorities should address these areas jointly. The management of occupational risk in the veterinary practice is a very important issue. Veterinary personnel should always wear protective equipment appropriate for all situations. As much as possible injuries through needles should be avoided as this may serve as portal of entry for pathogens (Weese and Jack, 2008). Client's education about issues such as the importance of vaccinating animals against zoonotic diseases such as rabies, control of endo and ectoparasites, as well as bite prevention will also help protect the community against zoonoses (NASPHV, 2010). Clients can also be educated by veterinary personnel of the possible risk of contacting zoonoses through ingestion of raw or undercook animal products such as milk (e.g. Salmnonelosis, Campylobacteriosis, brucellosis) and beef (bovine tuberculosis, cysticercosis). Clients with immunocompromised conditions, who insist on keeping their companion pets for psychologic benefits, can have them regularly check for zoonotic diseases to ensure that they do not pose any threat to them (Grant and Olsen, 1999). The role of veterinary meat inspection which targets public health can never be over emphasized in Bangladesh abattoirs. Routine disease surveillance and monitoring as well as disease reporting will help in providing disease records and identifying outbreaks immediately. Veterinarians can also pass information to the physicians when new diseases that have the potentials of infecting human are identified. Wound infections and abscesses can be caused by many zoonotic pathogens (Meyers et al., 2008). Therefore, veterinarians should wear protective outerwear and gloves during debridement, treatment, and bond aging of wounds. Immunization against known zoonoses is mandatory. Practicing veterinary personnel in Bangladesh who have contact with animals should receive pre-exposure rabies vaccination and antibody titer assessed every two years. They should also receive routine tetanus vaccination every 10 years (CDC, 2006a). Other roles may include development and production of biologicals, the control of animal populations which may serve as reservoirs of zoonotic pathogens, participation in outbreak investigations, prevention and control of food-borne illnesses of animal origin, and participation in environmental activities including vector, water, wildlife, and use of animal monitors. Roles and activities of public health veterinarians can be diverse but veterinarian should concentrate their focus on those issues impacting human and animal health.

The vast majority of zoonotic diseases in humans, as well as potential zoonotic diseases that companion animals are capable of transmitting are documented (Bharwana et al., 2012). Since zoonoses are diseases of animals that can infect humans, veterinarians, physicians, and public health officials need to work more closely together to control, prevent, and understand them. The veterinary profession contributes to improvement of human health by advancing biomedical and comparative medical research, preventing and addressing zoonotic diseases and enhancing environmental and ecosystem health as well. The veterinarian possesses qualities which can be directed to the investigation and management of human diseases and, although primarily trained to deal with diseases of lower animals, has a herd or population approach to the practice of medicine (Schwabe, 1986). The veterinarian must recognise and quarantine infected animals both within herds and at borders where animals are imported (Bharwana et al., 2012). Municipalities and city corporations are entrusted with carrying out meat inspection under their jurisdiction. However, the veterinary surgeons are generally inadequately trained in 


\section{Ariful Islam}

veterinary public health and food hygiene. There is no proper regulatory framework, which should address sanitary and safety issues. For other foods of animal origin such as milk, meat, eggs, and their value-added products, the sanitary inspectors of the health department have the authority of inspection and examination of such products. Therefore, marketing of products that pose the threat of spreading diseases in human. Thus, the public health inspection teal should include trained veterinarian to supervise animal origin foods and food products. But they are neither properly trained nor supervised by veterinarian. Throughout the world Veterinarians have been employed as policy makers and directors of public health programs in government departments and public health institute including international agencies such as the World Health Organization (WHO), the Food and Agriculture Organization (FAO) and the World Organization for Animal Health (OIE). Export of animal products needs to follow the World health Organization standards. In the near future, the role of international organizations is likely to intensify in monitoring regional diseases, in which Bangladesh should be prepared to participate.

\section{One Health approach}

The introduction of the concept of 'One Health' which takes a holistic approach to address human, animal, and ecosystem health, again emphasizes the role of a veterinarian as a leader in present society by addressing the risk and emergence of zoonotic diseases and promoting basic health care needs of the world. The One Health approach aims to enhance global efforts to mitigate and counter the emergence of zoonoses and other diseases. The approach calls for strong multisectoral and multidisciplinary collaboration, which moves beyond the strengthening of veterinary-public health systems to more clearly encompass disease prevention, with greater emphasis on safer food production, distribution and marketing practices, and adoption of sustainable animal agriculture and natural resource management (Papadopoulosa and Wilmera, 2011). It includes research on prevention, control, diagnosis, and treatment of diseases of animals on the basic biology, welfare, and care of animals. It is broad in scope and truly multidisciplinary and focuses on agro-and bio-terrorism, agriculture and animals sciences, antimicrobial resistance, basic and translational research, biomedical research, clinical medicine, combating existing and emerging diseases and zoonoses, comparative medicine, consumer support, diagnosis, surveillance, control, response and recovery directed at natural orientational threats that are chemical, toxicological, or radiological in nature, entomology, ethics, food safety security, global food and water systems, global trade and commerce, health communications, health of the environment and environmental preservation, implications of climate change, infectious disease ecology, integrated system for diseases diagnosis, land use and production systems and practice, mental health, microbiology education, occupational health, public awareness and public communications, public health and public policy, regulatory enforcement, scientific discovery and knowledge creation, support of biodiversity, training, veterinary and environmental health organizations and wildlife protection (AVMA, 2010).

One Health is very much an overarching concept that spans many disciplines, professions, and areas of interest that includes the protection of animal health, the relief of animal suffering, the conservation of livestock resources, the promotion of public health, and the advancement of medical knowledge. Close cooperation and interaction between veterinarians, occupational health physicians and public health operators is thus necessary, for a worldwide strategy to expand interdisciplinary collaborations and communications in all aspects of health care for humans, animals and the environment. This is what the One Health Approach is intended to be (Rabozzi et al., 2012).

Veterinary medicine is approximately one-tenth the size of the human medical profession and its ability to be optionally and simultaneously successful in areas such as biomedical research, agro-terrorism, food safety and security, and public health is challenging at best. Even though, incorporating One Health into their existing specialities is considered daunting, local human and veterinary medical associations can meet more frequently to discuss topics of mutual interest in their communities, under the common banner of One Health and can catalyze the concept to trickle down to clinicians at the local levels. Veterinarians are well grounded in population health, comparative medicine, and preventive medicine. The concept of One Health has become a rallying call in response to the failing health care need of our world and lack of collaborative effort of our veterinary and human professionals who in turn are focused over individualized health care and explore deeper into biomedical research have made the situation even worse. 
Zoonoses and role of Veterinarian in Bangladesh

\section{Zoonotic diseases and poverty in Bangladesh}

Veterinary public health, food hygiene and zoonoses control are essential to improve the health status of the people. Poverty is thought to be one of the risk factors for zoonoses and food-borne diseases in both rural and urban consumers. In addition, natural calamities and disease epidemics are frequent phenomena that particularly affect the poor people. However, as a developing country, Bangladesh does not have any emergency preparedness plan to combat such adverse situations. Neglected Veterinary medical profession and its extension services, poor people without any knowledge of zoonotic diseases who are in close contact with livestock and their products, and unhygienic processing, maintaining and marketing the livestock and livestock products have made the situation graver in Bangladesh. However, controls of zoonotic diseases which are transmissible from animal to man require ability and willingness for intersectoral and interinstitutional cooperation for all zoonoses control activities. Therefore, according to Samad (2011), the poor people in particular may tend to be at risk from zoonotic diseases for a number of reasons: (a) The poor often live in close contact with their livestock and are thus at increased risk of contracting zoonotic diseases, (b) Access to education on the prevention of zoonotic diseases in often unavailable to the poor livestock keepers, (c) Poor consumers may be at greater risk from foodborne diseases, (d) Informal sales of animal products make the maintenance of hygienic conditions and the control of food quality very difficult, (e) In poor and remote areas, hygienic abattoirs and facilities for meat inspection are often limited. Contracting a zoonotic disease may have serious consequences for a poor person who will probably have poor access to healthcare and can ill afford to have his/her ability to work impaired by illness. Multidisciplinary outbreak investigations can be an important tool in exploring novel characteristics and risk factors of emerging infectious zoonotic diseases in resource-poor settings in Bangladesh.

\section{CONCLUSION}

To confront the threats of emerging zoonotic diseases, much can be done by education, and in particular by growing the awareness of different health professionals, and facilitating communication and collaboration between veterinary, public health, agricultural and national policy making personnel. This will help us to approach and control zoonotic diseases in as efficient and effective a way as possible. There should be enhanced communication between veterinary, health and environmental services as well as community associates to secure exchange of relevant epidemiological information and reports on prevention and control activities for zoonotic diseases. The successful control of zoonoses is impossible without acceptable standards of life and social welfare in developing country like Bangladesh. Veterinary public health may, therefore, be considered as an indicator of the nation-wide of social well-being in a country.

\section{REFERENCES}

1. American Veterinary Medicine Association USA (2010). Available fromhttps://www.avma.org/Pages/home.aspx

2. Bharwana SA, Ali S, Abbas F and Baloch IH (2012). Risks of zoonoses in veterinarians and infection control practices. Greener Journal: Biological Science 2 (1): 7-12.

3. Centre for Disease Control (2006a). Preventing tetanus, diphtheria, and pertussis among adults: use of tetanus toxoid, reduced diphtheria toxoid and acellular pertussis vaccine. Recommendations of the Advisory Committee on Immunization Practices (ACIP). MMWR Recommendation and Reports 55: 1-37.

4. Dvorak G, Rovid-Spickler A and Roth JA (2008). Fast facts for zoonotic diseases of companion animals. In: Handbook for zoonotic diseases of companion animals. Ames, Iowa: Center for Food Security and Public Health, Iowa State University College of Veterinary Medicine, 284- 336.

5. Food and Agriculture Organization (2004). The pro-poor livestock policy initiatives: A living from Livestock Rome.

6. Grant S and Olsen CW (1999). Preventing zoonotic diseases in immunocompromised persons: The role of physicians and veterinarians. Emerging Infectious Diseases 5 (1): 159-163.

7. Lipton BA, Hopkins SG, Koehler JE and Digiacomo RF (2008). A survey of veterinarian involvement in zoonotic disease prevention practices. Journal of the American Veterinary Medical Association 233: 1242- 1249.

8. Meyers B, Schoeman JP, Goddard A and Picardi J (2008). The bacteriology and antimicrobial susceptibility of infected and non-infected dog bite wounds: fifty cases. Veterinary Microbiology 127: 360-368. 
9. Molyneux D, Hallaj Z, Keusch GT, McManus DP, Ngowi H, Cleaveland S, Ramos-Jimenez P, Gotuzzo E, Kar K, Sanchez A, Garba A, Carabin H, Bassili A, Chaignat CL, Meslin F, Abushama HM, Willingham AL and Kioy D (2011). Zoonoses and marginalised infectious diseases of poverty: Where do we stand? Parasites and Vectors 4: 106.

10. National Association of State Public Health Veterinarians (2010). Compendium for veterinary standard precautions for zoonotic disease prevention in veterinary personnel. Journal of the American Veterinary Medical Association 237 (12): 1403-1422.

11. Papadopoulosa A, Wilmera S (2011). One Health: A Primer. National Collaborating Centre for environmental Health.

12. Rabozzi G, Bonizzi L, Crespi E, Somaruga C, Sokooti M, Tabibi R, Vellere F, Brambilla G, Colosio C (2012). Emerging Zoonoses: the "One Health Approach". Saf Health Work, 3(1): 77-83.

13. Rahman AKMA, Berkvens D, Fretin D, Saegerma, C, Ahmed MU, Muhammad N, Hossain A, Abatih E (2012). Seroprevalence andrisk factors for brucellosis in a high-risk group of individuals in Bangladesh. Foodborne Pathogend and Disease, 9(3): 190-197.

14. Rahman MA (2014). Prevalence of Q-fever in domestic ruminants in Bangladesh, (MS thesis June 2014). Department of Medicine, BAU, Mymensingh-2202, Bangladesh.

15. Samad MA (2011). Public health threat caused by zoonotic diseases in Bangladesh, Bangladesh Journal of Veterinary Medicine 9 (2): 95 - 120

16. Schwabe CW (1986). Current status of hydatid disease: a zoonosis of increasing importance. In R.C.A. Thompson, ed. The biology of Echinococcus and hydatid disease, London, UK, George Allen \& Unwin, 81-113.

17. Weese JS and Jack DC (2008). Needlestick injuries in veterinary medicine. Canadian Veterinary Journal 49: 780 784.

18. World Health Organization (2006). The Control of Neglected Zoonotic Diseases: A route to poverty alleviation Geneva. Report of a joint WHO/DFID-AHP meetings with the participation of FAO and OIE, Geneva, 20 and 21 September 2005. Geneva: WHO. 